\title{
Genetic and phenotypic analysis of B-cell post-transplant lymphoproliferative disorders provides insights into disease biology
}

\author{
Efsevia Vakiani', Katia Basso², Ulf Klein², Mahesh M. Mansukhani', Gopeshwar Narayan², Paula M. Smith², \\ Vundavalli V. Murty ${ }^{1,2}$, Riccardo Dalla-Favera ${ }^{1,2}$, Laura Pasqualucci,2 and Govind Bhagat ${ }^{1 *}$ \\ 'Department of Pathology, Columbia University, New York, NY 10032, USA \\ ${ }^{2}$ Institute for Cancer Genetics, Columbia University, New York, NY 10032, USA
}

*Correspondence to: Dr Govind Bhagat, Department of Pathology, College of Physicians and Surgeons, Columbia University, VCI4-228, 630W 168th street, New York, NY 10032, USA.

E-mail: gb96@columbia.edu

Received: 3 February 2008 Revised: 16 March 2008

Accepted: 27 March 2008

\begin{abstract}
B-cell post-transplant lymphoproliferative disorders (PTLD) are classified as early lesions, polymorphic lymphomas (P-PTLD) and monomorphic lymphomas (M-PTLD). These morphologic categories are thought to reflect a biologic continuum, although supporting genetic data are lacking. To gain better insights into PTLD pathogenesis, we characterized the phenotypes, immunoglobulin (Ig) gene alterations and non-Ig gene (BCL6, RhoH/TTF, c-MYC, PAX5, CIITA, BCL7A, PIM1) mutations of 21 PTLD, including an IM-like lesion, 8 P-PTLD and 12 M-PTLD. Gene expression profile analysis was also performed in 12 cases. All PTLD with clonal Ig rearrangements showed evidence of germinal centre (GC) transit based on the analysis of Ig and BCL6 gene mutations, and $74 \%$ had a non-GC phenotype (BCL6 \pm MUM1 + ). Although surface Ig abnormalities were seen in 6/19 (32\%) PTLD, only three showed 'crippling' Ig mutations indicating other etiologies for loss of the B-cell receptor. Aberrant somatic hypermutation (ASHM) was almost exclusively observed in M-PTLD (8/12 vs. 1/8 P-PTLD) and all three recurrent cases analysed showed additional mutations in genes targeted by ASHM. Gene expression analysis showed distinct clustering of PTLD compared to B-cell non-Hodgkin lymphomas (B-NHL) without segregation of P-PTLD from non-GC M-PTLD or EBV + from EBV - PTLD. The gene expression pattern of PTLD appeared more related to that of memory and activated B-cells. Together, our results suggest that PTLD represent a distinct type of $\mathrm{B}-\mathrm{NHL}$ deriving from an antigen experienced B-cell, whose evolution is associated with accrual of genetic lesions. Copyright (C) 2008 John Wiley \& Sons, Ltd.
\end{abstract}

Keywords: post-transplant lymphoproliferative disorder; gene expression profile; aberrant somatic hypermutation; phenotype

\section{Introduction}

Post-transplant lymphoproliferative disorders (PTLD) encompass a heterogeneous group of lymphocytic proliferations, the vast majority of which are of B-cell origin. The current WHO classification categorizes them as early lesions, polymorphic lymphomas (P-PTLD) and monomorphic lymphomas (M-PTLD). Virtually, all early lesions are polyclonal and they appear to lack any known molecular alterations [1]. Many P-PTLD and most M-PTLD are clonal [1,2]. Compared to P-PTLD, M-PTLD harbour more karyotypic abnormalities, have a higher frequency of alterations of protooncogenes and carry mutations in a number of tumour-suppressor genes $[1,3,4]$. Mutations in genes due to aberrant somatic hypermutation (ASHM), a recently identified B-cell specific mechanism of genetic instability, have been reported to occur exclusively in M-PTLD [5,6]. The presence of distinct as well as shared cytogenetic aberrations among P-PTLD and M-PTLD [4] suggests that certain M-PTLD might arise as independent primary neoplasms, while others may represent progression of P-PTLD. The relationship of P-PTLD to M-PTLD, however, remains unknown.

Our understanding of the cellular origin of PTLD also remains unclear. A limited number of studies have shown that the vast majority of cases bear an imprint of germinal centre (GC) transit, as evidenced by the presence of somatically mutated immunoglobulin ( $\mathrm{Ig}$ ) genes, and most have non-GC immunophenotypes (BCL6 \pm MUM1+) [2,7,8]. However, more sensitive approaches, such as gene expression profiling, which have significantly advanced our knowledge of B-cell non-Hodgkin lymphoma (B-NHL) biology, have not been systematically applied to the study of PTLD.

The pathogenesis of PTLD is commonly associated with Epstein-Barr virus (EBV) infection, although in more recent studies $23-42 \%$ of PTLD have been reported to be EBV - [2,9]. It has been proposed that EBV - PTLD may be similar to B-NHL of immunocompetent hosts [10]; yet, a number of them respond to decreased immunosuppression, suggesting that immune deregulation plays a role in 
the pathogenesis of at least a subset of EBV- PTLD [9]. A minority of PTLD also lack surface B-cell receptor (BCR) and it is speculated that EBV-encoded proteins can substitute for BCR signalling and survival of the abnormal clones [11]. The relationship of EBV- PTLD to EBV+ PTLD or B-NHL occurring in immunocompetent individuals is not known at present.

To gain better insights into the pathogenesis of PTLD, we performed comprehensive phenotypic and genetic characterization of 21 PTLD. Gene expression profiles of a subset of cases were also analysed and compared to other types of B-NHL and cells representing normal B-cell maturational stages.

\section{Materials and methods}

\section{Case selection and morphologic analysis}

We searched our departmental database to identify PTLD for which frozen tissue samples were available. Frozen sections were reviewed prior to and after obtaining tissue for molecular studies, to assess tumour content and ensure morphologic representation, and only cases with $>90 \%$ lesional composition were used. Sections of formalin-fixed, paraffin-embedded tissue from the corresponding cases were stained with haematoxylin and eosin for morphologic review. PTLD were classified according to the current WHO criteria [12]. This study was approved by the Institutional Review Board of Columbia University.

\section{Immunohistochemistry, in situ hybridization and flow cytometry}

Immunohistochemical (IHC) stains were performed on paraffin-embedded tissue sections after moist heat-induced antigen retrieval, using an autostainer (Universal staining system, DAKO, Carpinteria, CA) and the primary antibodies listed in Table 2. The Envision Plus kit (DAKO) was used for detection. Staining for BLIMP-1 and activation-induced deaminase (AID) was performed as previously described $[13,14]$.

Cases were scored as positive if $>20 \%$ cells expressed the analysed antigen, except for LMP1, EBNA2 and BZLF1, where percentage of cellular expression was recorded.

In situ hybridization for EBV-encoded small RNAs (EBER) was performed using the manufacturer's protocol (INFORM EBER, Ventana, Tucson, AZ).

Three or four colour flow cytometric analysis (FACScan; BD Biosciences, San Diego, CA) was performed using a comprehensive panel of antibodies against B- and T-cell antigens, including $\operatorname{Ig}$ light $\left(\operatorname{Ig}_{\mathrm{L}}\right)$ and heavy chains $\left(\operatorname{Ig}_{\mathrm{H}}\right)$, according to standard methods. Data were analysed using the Cell Quest software (BD Biosciences).

\section{DNA and RNA extraction}

Genomic DNA and total RNA were extracted from frozen tissue according to standard methods. In all PTLD and in selected matched non-neoplastic tissue specimens, DNA was also extracted from paraffin-embedded tissue using the QIAamp DNA mini Kit (QIAGEN, Valencia, CA).

\section{Analysis of $\mathrm{Ig} \mathrm{V}_{\mathrm{H}}$ genes}

PCR amplification of the Ig heavy chain variable region $\left(\mathrm{V}_{\mathrm{H}}\right)$ genes was performed using DNA extracted from frozen tissue initially with $\operatorname{IgV}_{\mathrm{H}}$ gene family specific $\left(\mathrm{V}_{\mathrm{H}} 1-6\right)$ primers that hybridize to framework region (FR) 1 sequences and an antisense $\mathrm{J}_{\mathrm{H}}$ primer mix. If no clonal $\mathrm{IgV}_{\mathrm{H}}$ gene rearrangement was obtained, DNA was amplified with $\mathrm{V}_{\mathrm{H}} 1,3,4$ primers that hybridize to the $\operatorname{IgV}_{\mathrm{H}}$ leader (L) region (sequences for all primers have been previously reported $[15,16])$. PCR products were purified and sequenced as described [17], and sequences were compared to the most homologous germline $\mathrm{V}_{\mathrm{H}}$ genes in the IMGT (https://imgt.cines.fr) and IgBLAST (http:// www.ncbi.nlm.nih.gov/igblast/) databases. The presence of $\mathrm{IgV}_{\mathrm{H}}$ rearrangements was also determined using FR3 primers and DNA extracted from paraffin-embedded tissue as previously described [18].

The ratio of replacement to silent mutations in the complementarity determining regions and FRs was analysed using the multinomial distribution model available at www-stat.stanford.edu/immunoglobulin [19]. Determination of antigen selection was based on the analysis of FR mutations since interpretation of complementarity determining region mutations is controversial [20].

\section{Analysis of BCL6, PIMI, PAX5, RhoH/TTF, c-MYC, CIITA and BCL7A}

The oligonucleotide sequences used for amplification of the BCL6, PIM1, PAX5, RhoH/TTF, c-MYC, CIITA and $B C L 7 A 5^{\prime}$ sequences and the genomic regions amplified are described in Supplemental Table 1. These regions correspond to the intervals previously shown to harbour $>95 \%$ of all BCL6 mutations detected in B-NHL [21] and $>90 \%$ of mutations affecting the remaining six genes in diffuse large B-cell lymphomas (DLBCL) [6] (L. P., unpublished data). Purified amplicons were sequenced directly as described [17] and compared to the corresponding germline sequences (Supplemental Table 1) as previously reported [6]. Nucleotide changes due to previously reported polymorphisms or present in paired normal DNA (where available) were excluded from the calculation of mutation frequencies [6].

\section{Generation of gene expression profiles and data analyses}

Gene expression profile analysis was performed on 12 PTLD (1 IM-like lesion, 5 P-PTLD and 6 M-PTLD). A case each of IM-like lesion and M-PTLD of GC phenotype were included for analysis; the former, since cellular composition of the interfollicular areas in such lesions is morphologically indistinguishable from P-PTLD, and the latter, to determine any overriding effect of the post-transplant status (effect of immunosuppressive medi- 


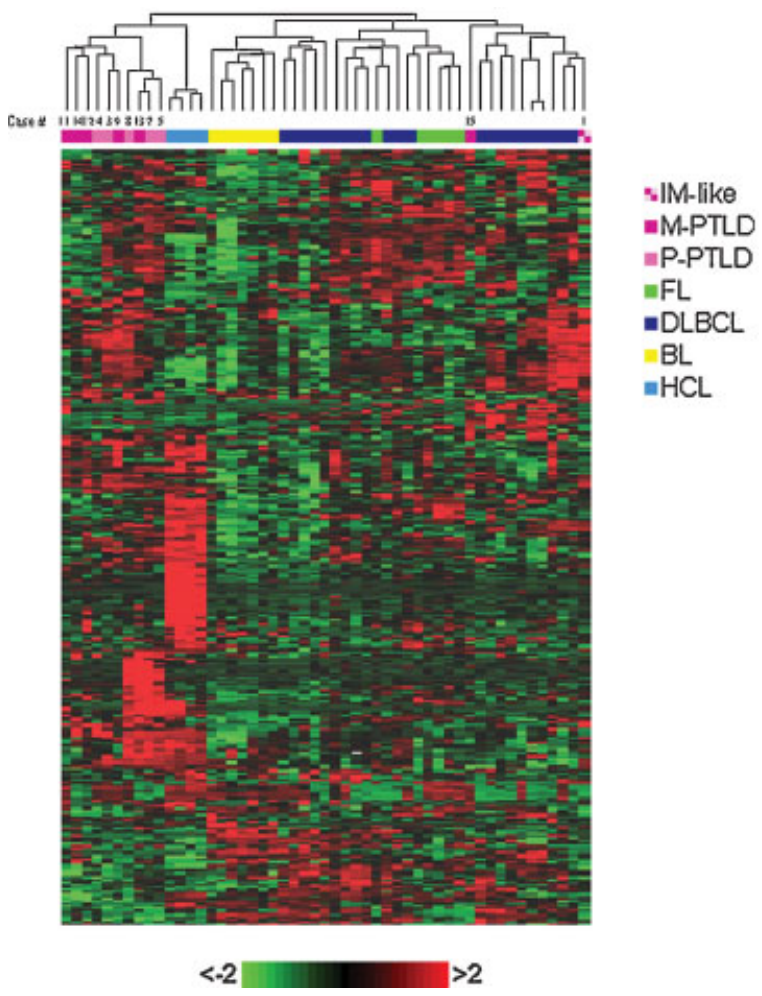

Figure I. Unsupervised hierarchical clustering performed on a representative panel of B-NHL, including 22 diffuse large B-cell lymphomas (DLBCL), 6 follicular lymphomas (FL), 7 Burkitt lymphomas (BL), 4 hairy cell leukaemias $(\mathrm{HCL})$ and 12 PTLD; 5 polymorphic (P-PTLD), 6 monomorphic (M-PTLD) and I IM-like lesion (case numbers shown correspond to PTLD in Table I). Clustering is based on the expression of 333 genes. (Colour scale identifies relative gene expression changes normalized by the standard deviation-zero representing the mean expression level of a given gene across the panel)

cations on the lymphoproliferations or their microenvironment) on the pattern of segregation of PTLD. Suboptimal RNA quality and insufficient numbers of distinct morphologic types of M-PTLD (e.g. BL) precluded analysis of the remaining cases. Complementary RNA was prepared as previously described and hybridized to HG-U95Av2 microarrays (Affymetrix, Santa Clara, CA) [22]. Gene expression values were determined by the GCOS 1.4 Microarray Suite 5.0 software (Affymetrix) using the Global Scaling option. In conjunction with previously generated expression profile data of other B-NHL subsets (derived from B-cells at different maturational stages), including EBV- DLBCL $(n=22 ; 14$ centroblastic and 8 immunoblastic), follicular lymphoma (FL, $n=6$ ), Burkitt lymphoma (BL, $n=7 ; 1 \mathrm{EBV}+$ and 6 EBV-), and hairy cell leukaemia (HCL, $n=4)$ ) [23,24], a hierarchical clustering algorithm based on the averagelinkage method was used to generate the dendrogram in Figure 1 [25]. Expression values of the selected genes were normalized to have a zero mean value and unit standard deviation, and the distance between two individual samples was calculated by Pearson distance analysis.

Supervised analysis was performed using the Genes@Work software platform [26]. PTLD gene expression profiles were compared to mantle cell lymphoma (MCL, $n=7)$, HCL $(n=8)$, chronic lymphocytic leukaemia (CLL, $n=10)$, FL $(n=6)$, BL $(n=7)$, DLBCL, including centroblastic $(n=15)$ and immunoblastic $(n=8)$ subtypes, and purified normal B-cell subsets (5 naïve, 5 memory and 10 GC) $[17,23,27]$. Cell type classification was performed as previously described [27]. Briefly, the classifier is a scoring function based on a set of differentially expressed genes between two phenotypes (higher score implies closer relatedness of the test sample to the phenotype set).

\section{Statistical analysis}

Fisher's exact test and Student's $t$-test were performed to evaluate differences between datasets consisting of categorical and numerical variables, respectively, and a $p$-value $<0.05$ was considered significant. Where applicable, the Spearman's rank correlation coefficient was also evaluated.

\section{Results}

\section{Patient characteristics}

Twenty-one PTLD from 18 patients (10M, 8F), including 3 recurrent PTLD, were studied (Table 1). Seven patients presented with nodal and 11 with extranodal disease. Median age at initial diagnosis was 21 years (mean 27.5 years, range 2-64 years) and the median time from transplant to initial diagnosis was 7.5 years (mean 6.0 years, range $0.3-17$ years). P-PTLD occurred significantly earlier than M-PTLD (3.1 vs. 8.7 years, $p=0.02)$. Six patients are currently alive $1.7-5$ years (median 2.6 years, mean 2.8 years) following initial presentation and 11 patients died 1 month-16 years (median 1.2 years, mean 2.9 years) post-diagnosis, 6 of PTLD and 5 of other causes. All three patients whose recurrent PTLD were analysed had variable durations of remissions between recurrences (1.2, 1.7 and 14 years).

\section{Morphologic and phenotypic analysis}

PTLD cases were classified as IM-like lesion $(n=1)$, P-PTLD $(n=8)$ and M-PTLD $(n=12)$. M-PTLD were further classified as DLBCL $(n=10)$ and BL $(n=2)$; the DLBCL were predominantly composed of centroblastlike cells. Morphologic features of the 12 PTLD used for gene expression profiling are shown in Supplemental Figure 1.

The results of IHC staining are shown in Table 2. The neoplastic cells in all 7 P-PTLD tested and in 7/10 (70\%) DLBCL were CD10-/BCL6 \pm /MUM1+/CD138-; these cases were classified as having a non-GC (post-GC) phenotype (Table 1) [28]. Three of 10 (30\%) DLBCL and both BL had a GC phenotype (CD10 \pm /BCL6+/MUM1-/ CD138-). Neoplastic cells in all PTLD expressed PAX5 and CD79a, and all except one were OCT2+; diminished $(n=4)$ or absent $(n=1)$ CD20 expression was seen in five cases (2/7, 29\% P-PTLD, 3/12, 25\% M-PTLD) by IHC staining and flow cytometry. One OCT2 + DLBCL lacked OCT2 expression on recurrence. One of 7 (14\%) P-PTLD and 9/12 $(75 \%)$ M-PTLD $(p=0.02)$ expressed PU.1. 


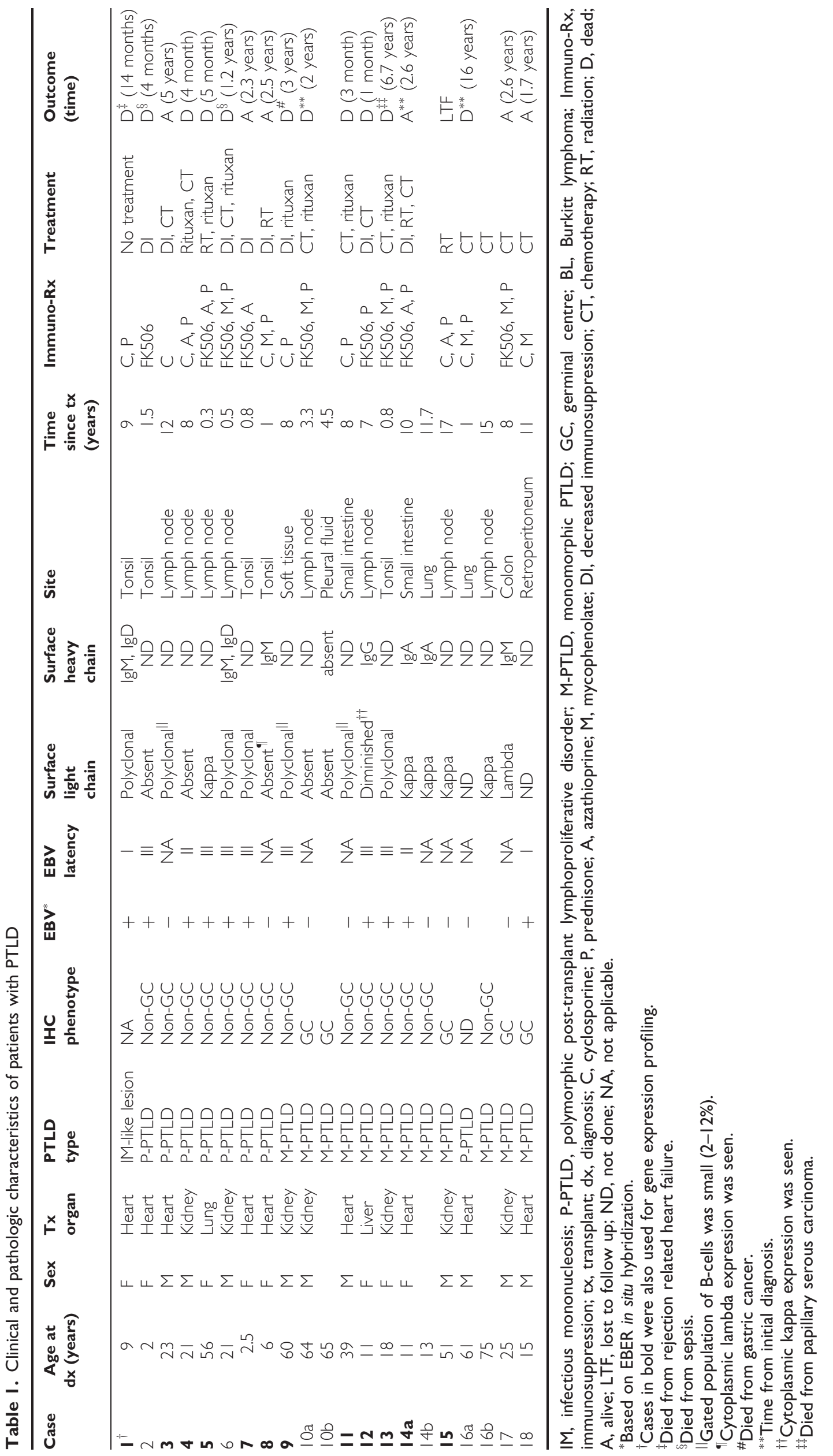


PTLD with a non-GC phenotype showed weak PU.1 staining, in contrast to PTLD with a GC phenotype (moderate to strong PU.1 staining), suggesting a correlation of PU.1 expression with GC phenotype, similar to DLBCL of immunocompetent individuals [29]. Neoplastic cells of 3/12 (25\%) M-PTLD expressed BLIMP-1, while only plasma cells expressed BLIMP-1 in P-PTLD. One DLBCL that was HLA-DR - by IHC (and dim HLA-DR+ by flow cytometry) had an aggressive course similar to that reported for HLA-DR- DLBCL of immunocompetent hosts [30]. Cytoplasmic staining for AID, an enzyme required for SHM and class switch recombination [31], was seen only in M-PTLD (0/7 vs. $10 / 12, p<0.005)$. Only a minority of nuclei in each case (rare-5\%) showed AID expression. There was no evidence of human herpes virus 8 infection as assessed with a stain for latency-associated nuclear antigen (LANA).

Flow cytometric assessment of $\operatorname{Ig}_{\mathrm{L}}$ and surface $\operatorname{Ig}_{\mathrm{H}}$ $\left(\mathrm{sIg}_{\mathrm{H}}\right)$ expression performed in 19 and 8 PTLD, respectively, demonstrated $\mathrm{Ig}_{\mathrm{L}}$ restriction in $8 / 19(42 \%)$ PTLD. A dominant $\operatorname{sIg}_{\mathrm{H}}$ was detected in 5/8 (63\%) PTLD. Absent or diminished $\operatorname{sIg}_{\mathrm{L}}$ expression was seen in $6 / 19$ (32\%) PTLD (3 P-PTLD, 3 M-PTLD); however, monotypic cytoplasmic light chains and $\operatorname{sIg}_{\mathrm{H}}$ were detected in 2 of these cases (Table 1).

\section{EBV infection}

Evidence of EBV infection (EBER+) was found in 11/21 (52\%) samples, including the IM-like lesion, 5/8 (63\%) P-PTLD, and 5/12 (42\%) M-PTLD (4/10 DLBCL, 1/2 BL). One EBER+ (and OCT2+) M-PTLD was EBER - (and OCT2-) on recurrence 1.7 years later. The types of viral latency are shown in Table 1. The IM-like lesion showed only rare (up to 5/HPF) scattered EBER+/LMP1-/ EBNA2 - cells (latency I), while 4/5 (80\%) P-PTLD and $3 / 5(60 \%)$ M-PTLD exhibited latency type-III infection (LMP1+/EBNA2+). The number of LMP1+ and EBNA2+ cells, however, varied significantly, indicating heterogeneity in latency types within a given PTLD, as shown previously [32]. One case each of a P-PTLD and M-PTLD had latency type-II (LMP-1+/EBNA2-) and the $\mathrm{EBER}+\mathrm{BL}$ had latency type-I infection. All $5 \mathrm{EBV}+$ P-PTLD and 2/5 (40\%) EBV+ M-PTLD showed variable BZLF1 (lytic phase protein) expression, similar to previous reports (Table 2) [32].

The majority of non-GC PTLD were EBV+ (9/14) and the majority of GC PTLD were EBV - (4/5); however, the correlation between phenotype and EBV infection did not reach statistical significance $(p=0.14)$. There was no significant difference in the mean time from transplant to initial presentation between EBV + and EBV - PTLD (5.2 vs. 7.2 years, $p=0.42$ ). AID expression was observed more frequently in EBV - PTLD, although the difference was not statistically significant (7/9 vs. $3 / 10, p=0.07)$.

\section{Analysis of $\lg \mathrm{V}_{\mathrm{H}}$ rearrangements and mutations}

Clonal $\operatorname{IgV}_{\mathrm{H}}$ gene rearrangements were detected in the majority of PTLD $(16 / 21,76 \%)$ and all except one harboured somatic mutations (mean frequency $9.2 \%$, range 3.7-19.3\%) (Table 3, Supplemental Table 2). The case lacking $\operatorname{IgV}_{\mathrm{H}}$ gene mutations had mutations in BCL6 (case \#7, Table 3). Overall, these findings indicate an origin of PTLD from GC experienced B-cells. There was no significant difference in the average $\operatorname{IgV}_{\mathrm{H}}$ mutation frequency between P-PTLD (7.7\%) and M-PTLD $(10.3 \%, p=0.38)$ or between EBV - PTLD (10.6\%) and $\mathrm{EBV}+\mathrm{PTLD}(7.5 \%, p=0.26)$.

Potentially functional Ig genes were predicted in 13 (81\%) of the 16 clonally rearranged PTLD (5/6 P-PTLD and 8/10 M-PTLD, Supplemental Table 2). The remaining three $(19 \%)$ cases displayed an original in-frame rearrangement that had acquired crippling mutations: one of these, an EBV+ P-PTLD, had a 295 (bp) deletion involving FR1 and FR2; two other samples, representing clonally related EBV - M-PTLD from the same patient, had a 1 bp deletion in the FR3 region, resulting in a frameshift with premature stop codon. All these three cases showed absent $\operatorname{sIg}_{\mathrm{L}}$ expression (and $\operatorname{sIg}_{\mathrm{H}}$ in one case evaluated) by flow cytometry. Notably, virtually all mutated functional rearrangements (11/12) showed lower than expected number of replacement FR mutations, indicating selection due to antigen pressure.

\section{Analysis of BCL6 mutations}

In agreement with previous studies, BCL6 mutations were seen in 14/21 (67\%) PTLD (Table 3) [2,3]. A total of 46 independent mutations were detected (mutations in $>1$ sample from the same patient were counted once), corresponding to an average mutation frequency of $0.11 / 100 \mathrm{bp}$ in the mutated cases (range, 0.06-0.45/ $100 \mathrm{bp})$. Of these, the majority were represented by single bp substitutions ( $n=43,18$ transitions, 25 transversions, ratio 0.72 ) and three were deletions.

No significant differences were observed between P-PTLD and M-PTLD with respect to total number of cases carrying BCL6 mutations $(5 / 8,63 \%$ vs. $9 / 12,75 \%$, $p=0.6)$ and average mutation frequency $(0.12 / 100 \mathrm{bp}$ vs. $0.35 / 100 \mathrm{bp}, p=0.19$ ). The presence (or frequency) of BCL6 mutations also did not correlate with the type of organ transplanted, immunosuppression, site of PTLD (nodal vs. extranodal), phenotype, EBV infection or AID expression. Contrary to a prior study [3], the presence of BCL6 mutations showed no correlation with clinical outcome. However, patients who died from disease had a significantly higher mutation frequency compared to those who are alive or died from other causes $(0.32 / 100 \mathrm{bp}$ vs. $0.05 / 100 \mathrm{bp}, p=0.03$ ).

\section{Analysis of genes targeted by ASHM}

To examine the role of ASHM in the pathogenesis and progression of PTLD, we sequenced the $5^{\prime}$ region of six non-Ig genes previously identified as ASHM targets, including two (CIITA and BCL7A) that have not been previously reported (L. P., unpublished observation). No mutations were seen in the IM-like lesion and this case was excluded from further analysis, since a clonal population 


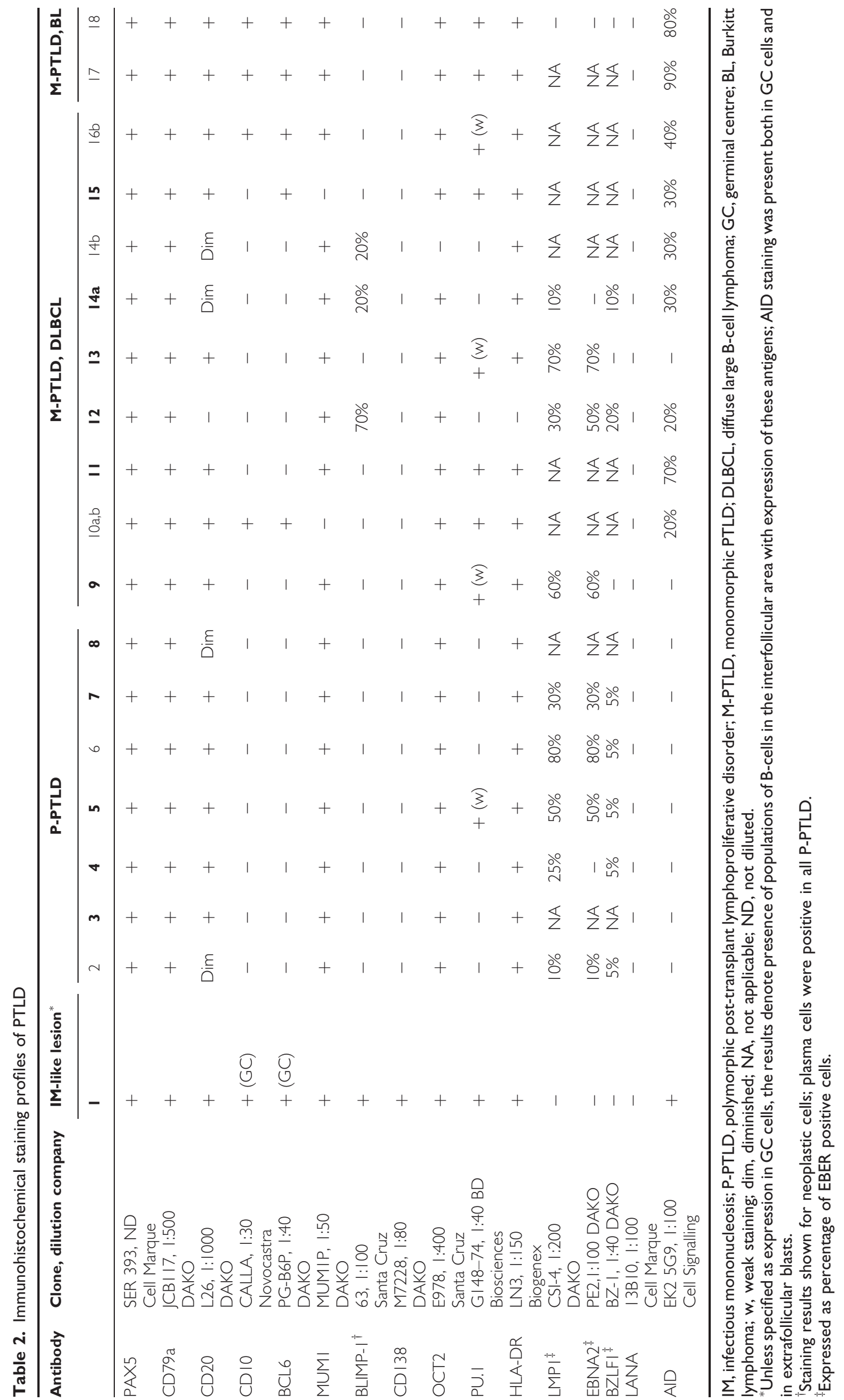




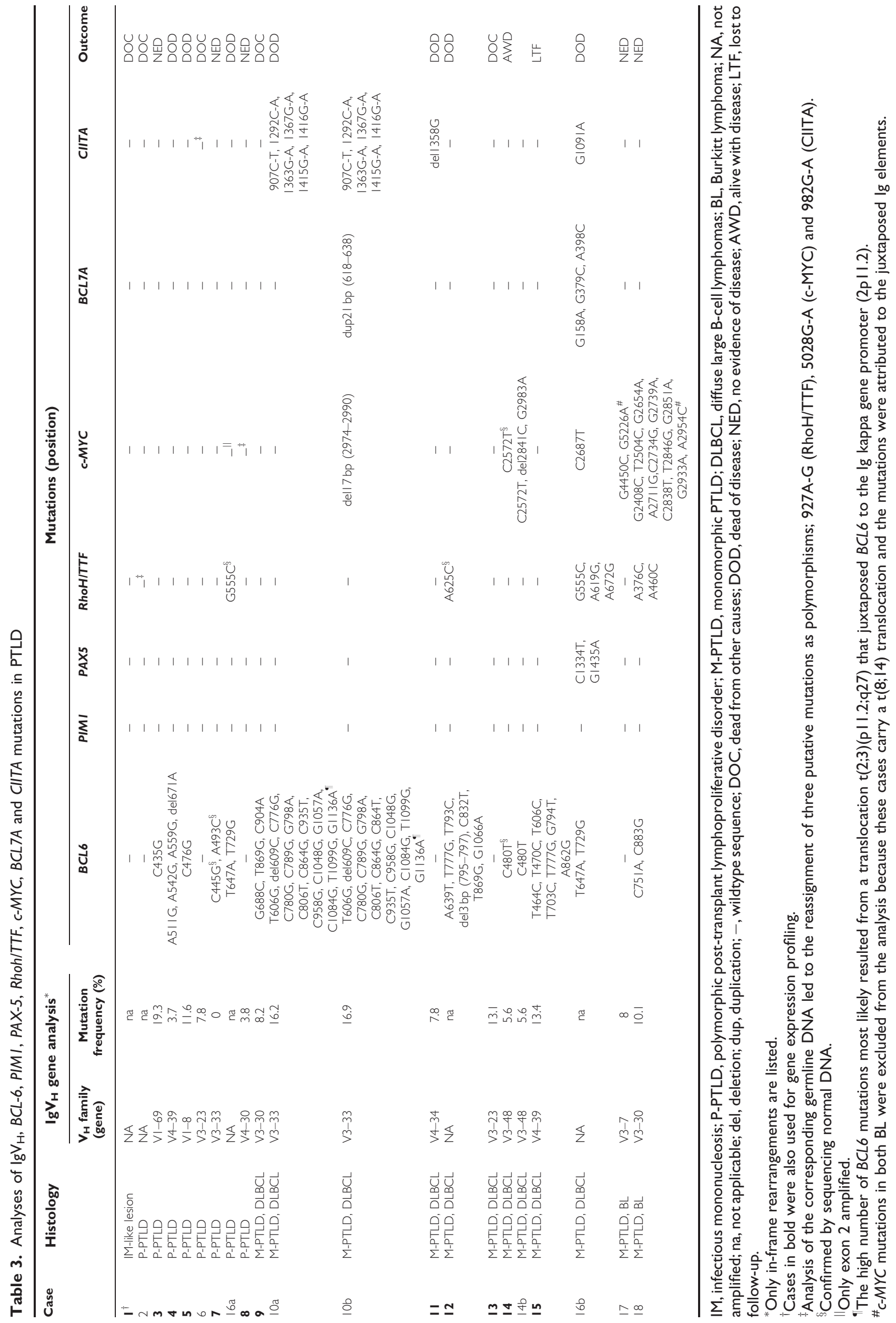


was not detected. Mutations affecting at least one gene were found in 9/20 (45\%) PTLD samples from six patients (Table 3). PAX5 was mutated in $1 / 20$ (5\%), RhoH/TTF in 4/ 20 (20\%), c-MYC in 4/18 (22\%), BCL7A in 2/20 (10\%) and CIITA in 4/20 (20\%) PTLD. No PIM1 mutations were observed. In total, 25 independent mutations were seen, consisting of 21 single bp substitutions (13 transitions, 8 transversions, ratio 1.63), three deletions and one duplication.

ASHM was significantly more frequent in M-PTLD than in P-PTLD (8/12 vs. 1/8 mutated cases, $p=0.028$ ), and correlated with AID expression (8/10 vs. $0 / 9, p<0.005)$. When considering EBV status, ASHM was observed more frequently in EBV - PTLD, both with regard to total number of cases (6/10 vs. $3 / 10, p=0.4)$ and mutation load (21 vs. 4 events, $p=0.05)$. No correlations were seen between ASHM and the type of organ transplanted, immunosuppression, site of PTLD or phenotype. Notably, all three recurrent PTLD acquired additional mutations in at least one gene, compared to the initial sample. Although, not statistically significant, a greater number of patients who died from disease had evidence of ASHM compared to those who died of other causes or are alive $(4 / 6,66.7 \%$ vs. $2 / 10,20 \%, p=0.1)$. The former also had a higher number of mutations (20 vs. 5 events, $p=0.07$ ), with similar mean follow up durations for the two groups (31.5 vs. 32.7 months, $p=0.96$ ).

\section{Gene expression profile analysis}

To investigate whether PTLD are characterized by a distinct gene expression signature, we compared gene expression profiles of 12 PTLD with 39 B-NHL samples representative of lymphomas of GC/post-GC derivation. Unsupervised hierarchical clustering showed that, with the exception of the M-PTLD with GC phenotype and the IM-like lesion, all PTLD segregated as a separate group (Figure 1). Additionally, PTLD expression profiles were also different from CLL, MCL [23,27] and marginal zone
B-cell lymphomas (GB, unpublished data). Thus, non-GC PTLD appear to represent a distinct type of B-NHL.

Supervised analysis, performed to identify genes differentially expressed between PTLD and normal or transformed B-cells, detected four genes whose expression levels were significantly higher in PTLD (PRDMI/ BLIMP-1, XBP-1, IFITM3 and PCMT1) and only one gene (C19ORF6), of unknown function, which was expressed at lower levels (Figure 2).

Both unsupervised and supervised analyses failed to distinguish P-PTLD from non-GC M-PTLD or EBV+ PTLD $(n=8)$ from EBV - PTLD $(n=4)$ (Figure 1 and data not shown).

Finally, in an attempt to better define the cell of origin of PTLD, we applied a classification procedure that measures the relatedness of PTLD gene expression profiles to those of normal B-cell subsets [27]. PTLD expression profiles were first compared to the gene expression signature that distinguishes GC (centroblasts and centrocytes) from non-GC (naïve and memory) B-cells. This analysis showed that PTLD are significantly more similar to non-GC B-cells (Figure 3A, E). On comparing PTLD with naïve and memory B-cells, PTLD appeared more related to memory B-cells (Figure 3B, E). Lastly, we evaluated the relationship of PTLD to memory B-cells versus EBV-immortalized lymphoblastoid cell lines (LCL), representative of activated B-cells, or multiple myeloma (MM) cell lines, representative of terminally differentiated plasma cells. In both analyses, the PTLD expression profile appeared significantly more similar to that of LCLs (Figure 3C-E).

\section{Discussion}

The present study was aimed at elucidating the molecular histogenesis of PTLD by performing a comprehensive immunophenotypic, genetic and genome-wide expression analysis on a panel of PTLD representative of the polymorphic and monomorphic subtypes. Our findings

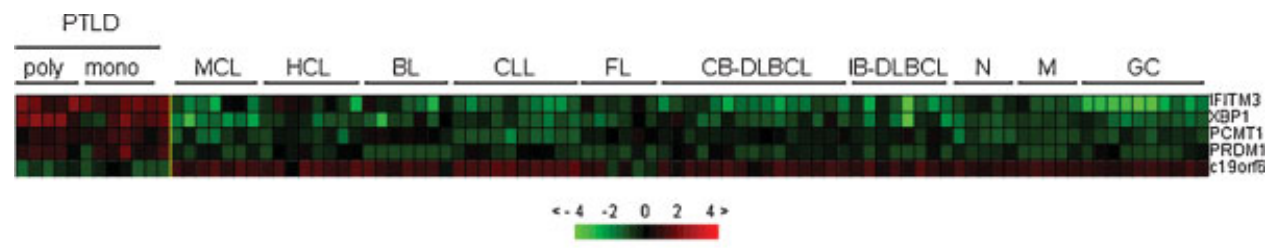

\begin{tabular}{|l|c|l|}
\hline Probe Set ID & Gene Symbol & Gene Tille \\
\hline $\mathbf{4 1 7 4 5}$ at & IFIMM3 & interferon induced transmembrane protein 3 (1-8U) \\
\hline 39755 at & XBP1 & X-box binding protein 1 \\
\hline 37737 at & PCMT1 & proten-L-isoaspartate(D-aspartate) O-melhyttransferase \\
\hline 31779 s at & PRDM1 & PR domain containing 1, with ZNF domain \\
\hline 41291 at & C19ar6 & chromosome 19 open reading frame 6 \\
\hline
\end{tabular}

Figure 2. Supervised analysis to identify differentially expressed genes between PTLD (5 polymorphic, 6 monomorphic and I IM-like lesion) and a representative panel of normal and neoplastic B-cells, which consists of 7 mantle cell lymphomas (MCL), 8 hairy cell leukaemias (HCL), 7 Burkitt lymphomas (BL), 10 chronic lymphocytic leukaemias (CLL), 6 follicular lymphomas (FL), 23 DLBCL (I5 centroblastic-CB-DLBCL, 8 immunoblastic - IB-DLBCL), naïve and memory B-cells (5 each), and I0 germinal centre B-cells. In the matrix, columns represent samples and rows correspond to genes. The scale bar provides visualization of difference in the z-scores (expression difference/standard deviation) relative to the mean. Genes are ranked based on the z-score (mean expression difference of the respective gene between phenotype and control group/standard deviation). Affymetrix probe set ID, gene symbol and title is provided for the differentially expressed genes 
A

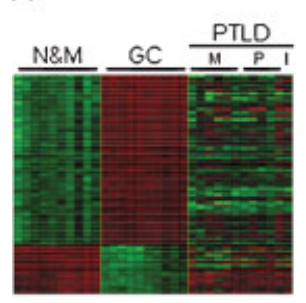

B

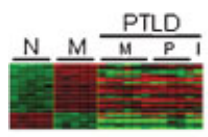

C

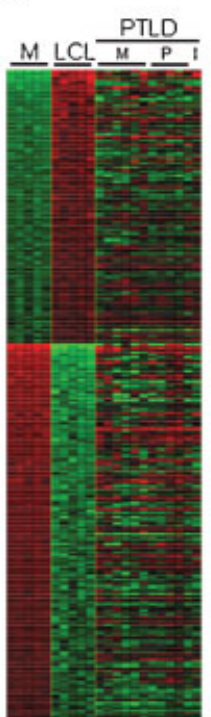

D

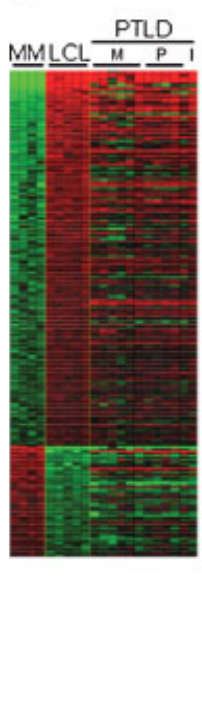

E

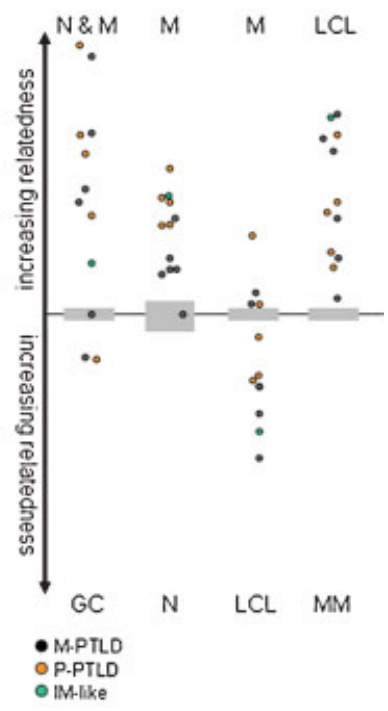

Figure 3. Supervised analysis to identify differentially expressed genes between two groups of samples: (A) naïve and memory B-cells (N\&M) compared to germinal centre (GC) B-cells; (B) naïve B-cells compared to memory B-cells; (C) EBV-transformed lymphoblastoid cell lines (LCL) compared to memory B-cells; (D) multiple myeloma cell lines (MM) compared to LCL. Expression of the selected genes investigated in PTLD is depicted on the right side of each matrix. In the matrices, each row represents a gene and each column represents a sample. Genes are ranked according to the z-score (mean expression difference of the gene between the phenotype group and the control group/standard deviation). The scale bar represents differences in the z-score relative to the mean. (E) Cell type classification to measure the relatedness of PTLD with the investigated populations. The grey area marks the $95 \%$ of confidence: $p$-value decreases with increasing distance from the $X$-axis

show that (1) non-GC PTLD are a homogeneous group of lymphoproliferations distinct from other B-NHL, as assessed by gene expression profiling; (2) the gene expression signature of non-GC PTLD is related to that of activated memory B-cells; (3) PTLD progression is associated with accrual of genetic lesions and (4) BCR abnormalities in PTLD have a multifactorial etiology.

\section{Non-GC PTLD are distinct from other B-NHL subtypes}

The relationship of PTLD to B-NHL of immunocompetent hosts is unknown. In fact, B-cell M-PTLD are morphologically indistinguishable from DLBCL (or BL), while P-PTLD do not resemble any currently recognizable type of B-NHL. Our comparative gene expression analysis of PTLD with other types of B-NHL demonstrates that non-GC PTLD have a homogeneous expression profile that differs from that of other types of B-NHL, including DLBCL (Figure 1). The histogenesis of GC PTLD could not be addressed by our study; however, the single GC PTLD in our series clustered with other GC type B-NHL of immunocompetent hosts. The observation that, on supervised analysis, PTLD only showed differential upregulation of transcription factors associated with plasma cell differentiation [33] suggests similarities with activated B-cell like DLBCL [34]. The DLBCL used in this study were not classified into cell-of-origin-based subgroups using a gene expression signature [34]. However, our unsupervised analysis included immunoblastic lymphomas, a subset of which are known to have phenotypes analogous to activated B-cell like DLBCL [35]. Thus, the unique clustering of PTLD most likely reflects distinct biologic features of these types of B-NHL.

PTLD also differ from DLBCL of immunocompetent hosts with regard to associated genetic lesions, specifically ASHM. The frequency of mutated post-transplant DLBCL was lower compared to DLBCL of immunocompetent hosts for all non-Ig genes except $c-M Y C$ and CIITA [6] (L. P. unpublished data), and all genes showed a lower average mutation frequency compared to de novo DLBCL [6]. Furthermore, no PIM1 mutations were observed, similar to an earlier report [5]. Overall, our data, in conjunction with previous findings in PTLD and HIV-associated B-NHL, suggest that immunosuppression-related lymphomas have lower frequencies of ASHM [5,36].

\section{Non-GC PTLD are related to activated memory B-cells}

Virtually, all clonal PTLD in our study appeared to arise from antigen selected B-cells and the majority (74\%) had non-GC phenotypes. These data extend the results of prior studies indicating derivation of PTLD from GC experienced B-cells, which have the phenotype of pre-terminally differentiated B-cells, that is BCL6+/-/MUM1+/ CD138- $[2,7,8]$. Of note, one P-PTLD lacked $\operatorname{IgV}_{\mathrm{H}}$ mutations, but had BCL6 mutations. The latter, in the absence of $\operatorname{IgV}_{\mathrm{H}}$ mutations, have previously been reported in other types of B-NHL, including PTLD [2]. This could reflect the stochastic nature of mutations targeting the two different loci by SHM [37], suggesting GC transit in the absence of Ig mutations. 
When compared to normal B-cell subsets, gene expression profiles of the non-GC PTLD were most similar to those of resting memory B-cells (Figure 3B, E). However, a comparison between PTLD, resting memory B-cells and LCLs showed a closer similarity of PTLD with LCLs (Figure 3C, E), which represent activated B-cells[38] and have expression profiles similar to IgM-activated B-cells [39]. Our findings, thus, support the hypothesis that non-GC PTLD, both EBV+ and EBV -, may derive from an activated memory B-cell. The suggestion that some PTLD are derived from memory B-cells was made by Timms et al. [8] based on the presence of Ig mutations with evidence of antigen selection. Previous studies had also pointed to similarities in the phenotypes and latent viral gene expression profiles between PTLD and LCLs or tumours developing in SCID mice inoculated with EBV infected human lymphocytes [40,41]. Since memory B-cells serve as reservoirs of latent EBV infection [42], it is conceivable that, in the setting of deregulated immune surveillance, activation or acquisition of growth advantage of cells within this compartment could result in a PTLD.

\section{Relationships between different types of PTLD}

Our results show that the gene expression programs of P-PTLD and non-GC M-PTLD are indistinguishable, as both unsupervised and supervised gene expression analyse could not segregate them into two distinct groups. This finding was somehow unexpected given the differences in morphology, cellular composition, and genetic abnormalities that characterize these two types of PTLD [1,3]. This is also the case with ASHM, which appears to be rare in P-PTLD but occurs in the majority of M-PTLD, as shown by our and a previous study [5]. One possible explanation for the inability of gene expression analysis to separate P-PTLD and M-PTLD is the existence of heterogeneity within one or both subtypes investigated. The observation that distinct or different combinations of genes are targeted by ASHM in different M-PTLD, suggests biologic differences among cases. However, an effect of subtle qualitative differences in cellular composition cannot be entirely excluded. If these assumptions are true, then a significantly larger survey will be required to identify distinct subsets within these two morphologic categories.

It has been hypothesized that PTLD progress along a continuum from polyclonal early lesions to monocloclonal M-PTLD [1,43]. The identification of cytogenetic aberrations common to both P-PTLD and M-PTLD supports this view [4]. However, a direct relationship between P-PTLD and M-PTLD has not been conclusively established. Only a few studies have evaluated clonal relatedness of PTLD, and clonal identity has been confirmed only in a handful of recurrent M-PTLD and one recurrent P-PTLD [1,43-45]. Our analysis provides evidence of a clonal relationship between a P-PTLD and a metachronous non-GC M-PTLD for the first time, supporting a precursor-product relationship between some P-PTLD and M-PTLD.

In our study, EBV + and EBV - PTLD did not show significant differences in gene expression profiles, suggesting a similar histogenesis of these PTLD subtypes. This result is in contrast to a recent study by Craig et al. who described differentially expressed genes, including $\mathrm{EBV}$ regulated transcripts, among $\mathrm{EBV}+$ and $\mathrm{EBV}-$ M-PTLD [46]. The differing results may be due to only a few cases analysed in both studies, differences in the types of cases evaluated or methodological differences in the biostatistical analyses. We performed a stringent analysis to identify only consistent differences among EBV + and EBV - PTLD, using both P-PTLD and M-PTLD. The gene expression differences among $\mathrm{EBV}+$ and $\mathrm{EBV}-$ cases identified by Craig et al. require validation by generating a classifier and testing them in an independent panel of cases, to demonstrate robustness of the expression differences. Clearly, larger series of PTLD of similar phenotypes need to be analysed to determine differences or similarities between $\mathrm{EBV}+$ and EBV - PTLD.

It also remains to be determined whether $\mathrm{EBV}+$ and EBV - PTLD share aberrations of signalling cascades, cell cycle regulators or proto-oncogenes, as has been suggested for classical Hodgkin lymphoma (cHL) [47]. The observation of an EBER + M-PTLD that recurred as a clonally related EBER - M-PTLD, raises the possibility that certain EBVPTLD may result from 'hit and run' oncogenesis [48], similar to $\mathrm{EBV}$ - sporadic BL and cHL [49,50]. A comprehensive survey to detect viral genomes in EBV - PTLD is required to definitively exclude a role of EBV or other viruses in the pathogenesis of such lymphoproliferations.

\section{Disease progression is associated with accrual of genetic lesions}

We could document accumulation of additional mutations by the neoplastic clone in three recurrent PTLD (including an M-PTLD representing progression of a prior P-PTLD). These findings might reflect clonal heterogeneity at inception, with survival of clones harbouring additional advantageous mutations, or may result from ongoing ASHM activity, as has been described for HIV-related B-NHL [36]. The correlation between presence of ASHM and AID expression by neoplastic cells makes the latter tenable. Our data, in conjunction with prior studies, suggest that progression of PTLD is associated with accrual of genetic lesions $[1,4,5]$. We speculate that a higher mutation frequency of non-Ig genes may connote recalcitrant disease, similar to observations in B-NHL of immunocompetent hosts [51]. However, larger series of recurrent PTLD need to be studied to evaluate the impact of ASHM on disease progression and prognosis.

\section{B-cell receptor abnormalities in PTLD have a multifactorial etiology}

Our analysis showed absent or reduced $\operatorname{sIg}_{\mathrm{L}}$ expression by flow cytometry in $32 \%$ of PTLD. While similar to earlier studies [52,53], a more recent report described a higher frequency of $\operatorname{sIg}_{\mathrm{L}}$ loss $(\approx 76 \%)$ based on IHC staining [54]. These discrepancies may be due to lower sensitivity of IHC-based methods compared to flow cytometry, as has been reported for other B-NHL [55]. 
Crippling $\operatorname{IgV}_{\mathrm{H}}$ gene mutations accounted for loss of $\operatorname{sIg}_{\mathrm{L}}$ in only half of $\mathrm{SIg}_{\mathrm{L}}-$ PTLD. In contrast to observations in cHL [56], no loss of OCT2 expression was seen in our $\mathrm{sIg}_{\mathrm{L}}-\mathrm{PTLD}$, in accordance with a recent report [57]. Absent PU.1 expression was seen in 4/6 (67\%) $\mathrm{sIg}_{\mathrm{L}}-\mathrm{PTLD}$, confirming the observations of a recent study [57]. This may be due to defective PU.1 expression, as proposed [57], or it may reflect activation of a plasma cell differentiation program [58]. Consistent with the latter hypothesis, three of our $\operatorname{sIg}_{L}-$ PTLD showed phenotypic evidence of transition to plasma cells, as evidenced by CD20 downregulation, cytoplasmic light chain expression, BLIMP-1 protein expression and/or CD43 expression (data not shown).

Capello et al. recently reported crippling mutations in $\operatorname{Ig}_{\mathrm{L}}$ genes [54]. Since we did not analyse mutations in $\operatorname{Ig}_{\mathrm{L}}$ genes, it is unclear whether cytoplasmic light chain expression, as observed in two of our $\operatorname{sIg}_{\mathrm{H}}+\mathrm{s} \mathrm{Ig}_{\mathrm{L}}-\mathrm{PTLD}$, is due to $\operatorname{Ig}_{\mathrm{L}}$ gene mutations or other aberrations. Expression of $\operatorname{sIg}_{\mathrm{H}}$ in the absence of $\operatorname{sIg}_{\mathrm{L}}$ has been reported in LCLs and certain types of B-NHL [59-61]. The etiology of this phenomenon appears multifactorial, some of the proposed mechanisms include truncations in the $\mathrm{V}_{\mathrm{H}}$ region secondary to abnormal splicing [59], posttranslational defects [62] and abnormal intracellular transport [63].

Irrespective of the mechanism of BCR loss, immunosuppression may allow the survival of BCR-deficient $\mathrm{B}$-cells, as detected in recipients of solid organ allografts [64]. In EBV + PTLD, the virus itself may rescue B-cell clones, which lack evidence of antigen selection or have crippling Ig mutations [2,7,8], a hypothesis supported by recent in vitro experiments [11]. However, the finding of sIg - EBV - PTLD by us and others [54] suggests that as yet unknown molecular alterations of the neoplastic cells may allow survival of EBV- BCR-deficient lymphocytes.

In summary, our findings indicate that non-GC PTLD are distinct from other B-NHL and appear closely related to activated memory B-cells. It is likely that distinct morphologic categories of PTLD are biologically heterogeneous. Further studies are required to explore the relationship of PTLD with other types of immunodeficiency-associated B-NHL.

\section{Acknowledgements}

GB would like to thank Dr Glauco Frizzera, formerly at Weill Cornell Medical Center, for morphologic review of PTLD and providing impetus for this study, and Dr Michael L. Shelanski for providing intramural funding.

\section{References}

1. Knowles DM, Cesarman E, Chadburn A, et al. Correlative morphologic and molecular genetic analysis demonstrates three distinct categories of posttransplantation lymphoproliferative disorders. Blood 1995; 85(2): 552-565.

2. Capello D, Cerri M, Muti G, et al. Molecular histogenesis of posttransplantation lymphoproliferative disorders. Blood 2003; 102(10): 3775-3785.

3. Cesarman E, Chadburn A, Liu YF, Migliazza A, Dalla-Favera R, Knowles DM. BCL-6 gene mutations in posttransplantation lymphoproliferative disorders predict response to therapy and clinical outcome. Blood 1998; 92(7): 2294-2302.

4. Vakiani E, Nandula SV, Subramaniyam S, et al. Cytogenetic analysis of B-cell posttransplant lymphoproliferations validates the World Health Organization classification and suggests inclusion of florid follicular hyperplasia as a precursor lesion. Hum Pathol 2007; 38(2): 315-325.

5. Cerri M, Capello D, Muti G, et al. Aberrant somatic hypermutation in post-transplant lymphoproliferative disorders. $\mathrm{Br} \mathrm{J} \mathrm{Hae}$ matol 2004; 127(3): 362-364.

6. Pasqualucci L, Neumeister P, Goossens T, et al. Hypermutation of multiple proto-oncogenes in B-cell diffuse large-cell lymphomas. Nature 2001; 412(6844): 341-346.

7. Brauninger A, Spieker T, Mottok A, Baur AS, Kuppers R, Hansmann ML. Epstein-Barr virus (EBV)-positive lymphoproliferations in post-transplant patients show immunoglobulin $\mathrm{V}$ gene mutation patterns suggesting interference of EBV with normal B cell differentiation processes. Eur J Immunol 2003; 33(6): 1593-1602.

8. Timms JM, Bell A, Flavell JR, et al. Target cells of EpsteinBarr-virus (EBV)-positive post-transplant lymphoproliferative disease: similarities to EBV-positive Hodgkin's lymphoma. Lancet 2003; 361(9353): 217-223.

9. Nelson BP, Nalesnik MA, Bahler DW, Locker J, Fung JJ, Swerdlow SH. Epstein-Barr virus-negative post-transplant lymphoproliferative disorders: a distinct entity? Am J Surg Pathol 2000; 24(3): 375-385.

10. Dotti G, Fiocchi R, Motta T, et al. Epstein-Barr virus-negative lymphoproliferate disorders in long-term survivors after heart, kidney, and liver transplant. Transplantation 2000; 69(5): 827-833.

11. Bechtel D, Kurth J, Unkel C, Kuppers R. Transformation of BCR-deficient germinal-center B cells by EBV supports a major role of the virus in the pathogenesis of Hodgkin and posttransplantation lymphomas. Blood 2005; 106(13): 4345-4350.

12. Harris N, Swerdlow S, Frizzera G, Knowles D. Post-transplant lymphoproliferative disorders. In Tumours of Haematopoietic and Lymphoid Tissues, Jaffe E, Harris N, Vardiman J (eds). International Agency for Research on Cancer: Lyon, 2001; 264-269.

13. Cattoretti G, Buttner M, Shaknovich R, Kremmer E, Alobeid B, Niedobitek G. Nuclear and cytoplasmic AID in extrafollicular and germinal center B cells. Blood 2006; 107(10): 3967-3975.

14. Cattoretti G, Angelin-Duclos C, Shaknovich R, Zhou H, Wang D, Alobeid B. PRDM1/Blimp-1 is expressed in human B-lymphocytes committed to the plasma cell lineage. J Pathol 2005; 206(1): 76-86.

15. Fais F, Gaidano G, Capello D, et al. Immunoglobulin V region gene use and structure suggest antigen selection in AIDS-related primary effusion lymphomas. Leukemia 1999; 13(7): 1093-1099.

16. Kuppers R, Rajewsky K, Hansmann ML. Diffuse large cell lymphomas are derived from mature $\mathrm{B}$ cells carrying $\mathrm{V}$ region genes with a high load of somatic mutation and evidence of selection for antibody expression. Eur J Immunol 1997; 27(6): 1398-1405.

17. Pasqualucci L, Migliazza A, Fracchiolla N, et al. BCL-6 mutations in normal germinal center B cells: evidence of somatic hypermutation acting outside Ig loci. Proc Natl Acad Sci USA 1998; 95(20): 11816-11821.

18. Sioutos N, Bagg A, Michaud GY, et al. Polymerase chain reaction versus Southern blot hybridization. Detection of immunoglobulin heavy-chain gene rearrangements. Diagn Mol Pathol 1995; 4(1): 8-13.

19. Lossos IS, Tibshirani R, Narasimhan B, Levy R. The inference of antigen selection on Ig genes. J Immunol 2000; 165(9): $5122-5126$.

20. Klein U, Goossens T, Fischer M, et al. Somatic hypermutation in normal and transformed human B cells. Immunol Rev 1998; 162: 261-280.

21. Migliazza A, Martinotti S, Chen W, et al. Frequent somatic hypermutation of the $5^{\prime}$ noncoding region of the BCL6 gene in B-cell lymphoma. Proc Natl Acad Sci USA 1995; 92(26): 12520-12524. 
22. Basso K, Klein U, Niu H, et al. Tracking CD40 signaling during germinal center development. Blood 2004; 104(13): 4088-4096.

23. Basso K, Liso A, Tiacci E, et al. Gene expression profiling of hairy cell leukemia reveals a phenotype related to memory B cells with altered expression of chemokine and adhesion receptors. J Exp Med 2004; 199(1): 59-68.

24. Klein U, Gloghini A, Gaidano G, et al. Gene expression profile analysis of AIDS-related primary effusion lymphoma (PEL) suggests a plasmablastic derivation and identifies PEL-specific transcripts. Blood 2003; 101(10): 4115-4121.

25. Eisen MB, Spellman PT, Brown PO, Botstein D. Cluster analysis and display of genome-wide expression patterns. Proc Natl Acad Sci USA 1998; 95(25): 14863-14868.

26. Lepre J, Rice JJ, Tu Y, Stolovitzky G. Genes@Work: an efficient algorithm for pattern discovery and multivariate feature selection in gene expression data. Bioinformatics 2004; 20(7): 1033-1044.

27. Klein U, Tu Y, Stolovitzky GA, et al. Gene expression profiling of B cell chronic lymphocytic leukemia reveals a homogeneous phenotype related to memory B cells. J Exp Med 2001; 194(11): 1625-1638.

28. Hans CP, Weisenburger DD, Greiner TC, et al. Confirmation of the molecular classification of diffuse large B-cell lymphoma by immunohistochemistry using a tissue microarray. Blood 2004; 103(1): 275-282.

29. Torlakovic E, Malecka A, Myklebust JH, et al. PU.1 protein expression has a positive linear association with protein expression of germinal centre B cell genes including BCL-6, CD10, CD20 and CD22: identification of PU.1 putative binding sites in the BCL-6 promotor. J Pathol 2005; 206(3): 312-319.

30. Rimsza LM, Roberts RA, Miller TP, et al. Loss of MHC class II gene and protein expression in diffuse large B-cell lymphoma is related to decreased tumor immunosurveillance and poor patient survival regardless of other prognostic factors: a follow-up study from the Leukemia and Lymphoma Molecular Profiling Project. Blood 2004; 103(11): 4251-4258.

31. Muramatsu M, Kinoshita K, Fagarasan S, Yamada S, Shinkai Y, Honjo T. Class switch recombination and hypermutation require activation-induced cytidine deaminase (AID), a potential RNA editing enzyme. Cell 2000; 102(5): 553-563.

32. Shaknovich R, Basso K, Bhagat G, et al. Identification of rare Epstein-Barr virus infected memory B cells and plasma cells in non-monomorphic post-transplant lymphoproliferative disorders and the signature of viral signaling. Haematologica 2006; 91(10): 1313-1320.

33. Shapiro-Shelef M, Calame K. Plasma cell differentiation and multiple myeloma. Curr Opin Immunol 2004; 16(2): 226-234.

34. Staudt LM, Dave S. The biology of human lymphoid malignancies revealed by gene expression profiling. Adv Immunol 2005; 87: 163-208.

35. Carbone A, Gloghini A, Larocca LM, et al. Expression profile of MUM1/IRF4, BCL-6, and CD138/syndecan-1 defines novel histogenetic subsets of human immunodeficiency virus-related lymphomas. Blood 2001; 97(3): 744-751.

36. Gaidano G, Pasqualucci L, Capello D, et al. Aberrant somatic hypermutation in multiple subtypes of AIDS-associated nonHodgkin lymphoma. Blood 2003; 102(5): 1833-1841.

37. Pasqualucci L, Neri A, Baldini L, Dalla-Favera R, Migliazza A. BCL-6 mutations are associated with immunoglobulin variable heavy chain mutations in B-cell chronic lymphocytic leukemia. Cancer Res 2000; 60(20): 5644-5648.

38. Young LS, Rickinson AB. Epstein-Barr virus: 40 years on. Nat Rev Cancer 2004; 4(10): 757-768.

39. Cahir-McFarland ED, Carter K, Rosenwald A, et al. Role of NF-kappa B in cell survival and transcription of latent membrane protein 1-expressing or Epstein-Barr virus latency III-infected cells. J Virol 2004; 78(8): 4108-4119.

40. Rowe M, Young LS, Crocker J, Stokes H, Henderson S, Rickinson $\mathrm{AB}$. Epstein-Barr virus (EBV)-associated lymphoproliferative disease in the SCID mouse model: implications for the pathogenesis of EBV-positive lymphomas in man. $J$ Exp Med 1991; 173(1): 147-158.
41. Thomas JA, Hotchin NA, Allday MJ, et al. Immunohistology of Epstein-Barr virus-associated antigens in B cell disorders from immunocompromised individuals. Transplantation 1990; 49(5): 944-953.

42. Babcock GJ, Decker LL, Volk M, Thorley-Lawson DA. EBV persistence in memory B cells in vivo. Immunity 1998; 9(3): 395-404.

43. Nalesnik MA, Jaffe R, Starzl TE, et al. The pathology of posttransplant lymphoproliferative disorders occurring in the setting of cyclosporine A-prednisone immunosuppression. Am J Pathol 1988; 133(1): 173-192.

44. Wu TT, Swerdlow SH, Locker J, et al. Recurrent Epstein-Barr virus-associated lesions in organ transplant recipients. Hum Pathol 1996; 27(2): 157-164.

45. Kaplan MA, Ferry JA, Harris NL, Jacobson JO. Clonal analysis of posttransplant lymphoproliferative disorders, using both episomal Epstein-Barr virus and immunoglobulin genes as markers. Am J Clin Pathol 1994; 101(5): 590-596.

46. Craig FE, Johnson LR, Harvey SA, et al. Gene expression profiling of Epstein-Barr virus-positive and -negative monomorphic B-cell posttransplant lymphoproliferative disorders. Diagn Mol Pathol 2007; 16(3): 158-168.

47. Knecht H, Berger C, McQuain C, et al. Latent membrane protein 1 associated signaling pathways are important in tumor cells of Epstein-Barr virus negative Hodgkin's disease. Oncogene 1999; 18(50): 7161-7167.

48. Ambinder RF. Gammaherpesviruses and "Hit-and-Run" oncogenesis. Am J Pathol 2000; 156(1): 1-3.

49. Gan YJ, Razzouk BI, Su T, Sixbey JW. A defective, rearranged Epstein-Barr virus genome in EBER-negative and EBERpositive Hodgkin's disease. Am J Pathol 2002; 160(3): 781-786.

50. Razzouk BI, Srinivas S, Sample CE, Singh V, Sixbey JW. Epstein-Barr Virus DNA recombination and loss in sporadic Burkitt's lymphoma. J Infect Dis 1996; 173(3): 529-535.

51. Rossi D, Berra E, Cerri M, et al. Aberrant somatic hypermutation in transformation of follicular lymphoma and chronic lymphocytic leukemia to diffuse large B-cell lymphoma. Haematologica 2006; 91(10): 1405-1409.

52. Dunphy CH, Gardner LJ, Grosso LE, Evans HL. Flow cytometric immunophenotyping in posttransplant lymphoproliferative disorders. Am J Clin Pathol 2002; 117(1): 24-28.

53. Kaleem Z, Hassan A, Pathan MH, White G. Flow cytometric evaluation of posttransplant B-cell lymphoproliferative disorders. Arch Pathol Lab Med 2004; 128(2): 181-186.

54. Capello D, Cerri M, Muti G, et al. Analysis of immunoglobulin heavy and light chain variable genes in post-transplant lymphoproliferative disorders. Hematol Oncol 2006; 24(4): 212-219.

55. Li S, Eshleman JR, Borowitz MJ. Lack of surface immunoglobulin light chain expression by flow cytometric immunophenotyping can help diagnose peripheral B-cell lymphoma. Am J Clin Pathol 2002; 118(2): 229-234.

56. Stein H, Marafioti T, Foss HD, et al. Down-regulation of BOB.1/ OBF. 1 and Oct2 in classical Hodgkin disease but not in lymphocyte predominant Hodgkin disease correlates with immunoglobulin transcription. Blood 2001; 97(2): 496-501.

57. Lucioni M, Capello D, Riboni R, et al. B-cell posttransplant lymphoproliferative disorders in heart and/or lungs recipients: clinical and molecular-histogenetic study of 17 cases from a single institution. Transplantation 2006; 82(8): 1013-1023.

58. Pettersson M, Sundstrom C, Nilsson K, Larsson LG. The hematopoietic transcription factor PU.1 is downregulated in human multiple myeloma cell lines. Blood 1995; 86(7): 2747-2753.

59. Cogne M, Silvain C, Khamlichi AA, Preud'homme JL. Structurally abnormal immunoglobulins in human immunoproliferative disorders. Blood 1992; 79(9): 2181-2195.

60. Hendershot L, Levitt D. Analysis of surface mu-chain expression in human lymphoblastoid cell lines that do not produce light chains. J Immunol 1984; 132(1): 502-509.

61. Kaleem Z, Zehnbauer BA, White G, Zutter MM. Lack of expression of surface immunoglobulin light chains in B-cell non-Hodgkin lymphomas. Am J Clin Pathol 2000; 113(3): 399-405. 
62. Vuillier F, Dumas G, Magnac C, et al. Lower levels of surface B-cell-receptor expression in chronic lymphocytic leukemia are associated with glycosylation and folding defects of the mu and CD79a chains. Blood 2005; 105(7): 2933-2940.

63. Lee BS, Alvarez X, Ishido S, Lackner AA, Jung JU. Inhibition of intracellular transport of B cell antigen receptor complexes by
Kaposi's sarcoma-associated herpesvirusK1. J Exp Med 2000; 192(1): 11-21.

64. Schauer E, Webber S, Green M, Rowe D. Surface immunoglobulin-deficient Epstein-Barr virus-infected B cells in the peripheral blood of pediatric solid-organ transplant recipients. J Clin Microbiol 2004; 42(12): 5802-5810. 